\title{
Linear response at criticality
}

\author{
Adam Svenkeson, ${ }^{1}$ Mauro Bologna, ${ }^{2}$ and Paolo Grigolini ${ }^{1}$ \\ ${ }^{1}$ Center for Nonlinear Science, University of North Texas, P.O. Box 311427, Denton, Texas 76203-1427, USA \\ ${ }^{2}$ Instituto de Alta Investigación, Universidad de Tarapacá-Casilla 6-D Arica, Chile
}

(Received 22 August 2012; published 24 October 2012)

\begin{abstract}
We study a set of cooperatively interacting units at criticality, and we prove with analytical and numerical arguments that they generate the same renewal non-Poisson intermittency as that produced by blinking quantum dots, thereby giving a stronger support to the results of earlier investigation. By analyzing how this out-ofequilibrium system responds to harmonic perturbations, we find that the response can be described only using a new form of linear response theory that accounts for aging and the nonergodic behavior of the underlying process. We connect the undamped response of the system at criticality to the decaying response predicted by the recently established nonergodic fluctuation-dissipation theorem for dichotomous processes using information about the second moment of the fluctuations. We demonstrate that over a wide range of perturbation frequencies the response of the cooperative system is greatest when at criticality.
\end{abstract}

DOI: 10.1103/PhysRevE.86.041145

PACS number(s): 05.40.Jc, 05.45.-a, 05.10.Gg

\section{INTRODUCTION}

The physics of blinking quantum dots (BQDs) [1-4] has generated a big interest but it is still a not yet fully understood phenomenon [2]. Analysis of the luminescence fluctuations led to the discovery $[5,6]$ that these fluctuations are non-Poisson renewal processes. In the special case where the time distance $\tau$ between two consecutive transitions from one to the other state is given by an inverse power law,

$$
\psi(\tau) \propto \frac{1}{\tau^{\mu}},
$$

with $\mu<2$, the process is a form of weak chaos [7] characterized by ergodicity breakdown, thereby requiring a generalization of some of the fundamental theoretical tools of statistical physics, the Khinchin theorem [8] and the fluctuation-dissipation theorem (FDT) $[9,10]$.

We note that the FDT, or, equivalently, the linear response (LR) of a system to an external perturbation is one of the major problems of modern physics [11]. Although the LR problem is fully solved in the case of physical systems at thermodynamical equilibrium, it is still the object of debate and controversies when applied to nonergodic physical systems. The perennial out-of-equilibrium condition of BQD fluorescence, in a striking conflict with the equilibrium condition hypothesized by the pioneer approach to LR [11], has led some authors to declare the death of LR [12]. More recently, the authors of Ref. [13] have shown that the irretrievable nonequilibrium nature of these processes generates a new form of LR.

It has to be pointed out that this problem is very difficult to solve because in addition to the nonergodic nature of these processes, the origin itself of this anomalous form of intermittence is not yet fully understood. The authors of Ref. [14] adopted the decision making (DM) model of Ref. [15] to explain the origin of BQD fluorescence intermittence, and although this interpretation is not shared by the advocates of the Auger effect [16] that seems to be much more widely accepted, we base this paper on the cooperative model of Ref. [15].
This choice is dictated by two main reasons. First, as we shall see, the adoption of this cooperative model generates a Markovian Liouville-like equation of motion for the nonergodic fluctuation, bypassing the problems met by the authors of Refs. [12,13]. In fact, the theoretical work of these authors rests only on the experimental information of the statistical properties of the "dark" and "light" states, which yields a fractional Fokker-Planck equation (FFP), a non-Markovian equation of motion, whose response to perturbation can only be expressed with an ambiguous Liouville-like equation and as such remains an open problem [17]. The adoption of the assumption that nonergodic intermittence is generated by the cooperative action of a very large number of units at criticality generates a nonlinear Langevin equation, whose positive and negative fluctuations around the vanishing mean value may be be interpreted as "light" and "dark" states, respectively. Focusing only on the nonergodic statistics of these "light" and "dark" states would lead us to the same FFP as that adopted by the researchers claiming either the LR death [12] or its extension [13] to the nonergodic condition. However, we shall address the issue of the response to perturbation directly on the global field, with no ambiguity whatsoever.

According to the nonergodic FDT [10], the response of the intermittent variable $\eta(t)$ to the harmonic perturbation $F(t)=$ $\epsilon \cos (\omega t)$ is given by

$$
\langle\eta(t)\rangle \propto R(t) \cos (\omega t+\phi)
$$

where $R(t)$ denotes the rate of crucial events, i.e., changes in state from "light" to "dark" or vice versa, whose decay in time is generated by the out-of-equilibrium preparation of the system at $t=0$. Note that when $\mu<2$,

$$
R(t) \propto \frac{1}{t^{2-\mu}}
$$

It is remarkable that in the long-time limit a complex system does not respond to harmonic perturbation, whereas it is proven to respond to stimuli with the same complexity [13].

There is a further good reason to use the cooperative model of Ref. [15] along with the assumption that the nonergodic nature of intermittency is a manifestation of criticality. This 
is the conviction that criticality, with its long-range correlation [18], may be the key condition for the origin of the intelligent behavior of cooperative systems, from a flock of birds $[19,20]$ to the human brain [21,22]. Thus, the linear response of a cooperative system at criticality, discussed in this paper, affords at the same time an interesting contribution to understanding how these complex systems may respond to external stimuli.

This paper is organized as follows. In Sec. II we introduce the DM model, approximating analytically its dynamic behavior in the all-to-all case. In Sec. III we use our approximation to study the intermittent properties of the fluctuations at criticality and find similarities to the intermittent behavior of BQDs. In Sec. IV A we adopt a nonstationary approach to LR to understand analytically how the out-of-equilibrium system responds to external perturbation. Section IV B considers the response of the system in the absence of fluctuations, with the benefit of recovering a form of the nonergodic FDT. Section VA is a numerical treatment of the response to harmonic perturbation and gives valuable information about the effect of criticality. In Sec. V B we connect the response in the case of the nonergodic FDT for dichotomous processes to the nonstationary LR for the nondichotomous fluctuations at criticality.

\section{DECISION MAKING MODEL AT CRITICALITY}

The DM model of Ref. [15] generates the cooperative dynamics of a set of network nodes, each of which is described by the master equation

$$
\begin{aligned}
& \frac{d}{d t} p_{1}^{(i)}(t)=-\frac{g_{12}^{(i)}(t)}{2} p_{1}^{(i)}(t)+\frac{g_{21}^{(i)}(t)}{2} p_{2}^{(i)}(t), \\
& \frac{d}{d t} p_{2}^{(i)}(t)=-\frac{g_{21}^{(i)}(t)}{2} p_{2}^{(i)}(t)+\frac{g_{12}^{(i)}(t)}{2} p_{1}^{(i)}(t),
\end{aligned}
$$

where

$$
g_{12}^{(i)} \equiv g_{0} \exp \left[K \frac{\left(N_{2}^{(i)}-N_{1}^{(i)}\right)}{N^{(i)}}\right]
$$

and

$$
g_{21}^{(i)} \equiv g_{0} \exp \left[K \frac{\left(N_{1}^{(i)}-N_{2}^{(i)}\right)}{N^{(i)}}\right]
$$

are the transition rates between the two possible states. The symbol $N^{(i)}$ denotes the number of nodes linked to the $i$ th node, with $N_{1}^{(i)}$ and $N_{2}^{(i)}$ being those in the first and second state, respectively. Of course, $N^{(i)}=N_{1}^{(i)}+N_{2}^{(i)}$. The control parameter $K$ is a measure of the cooperation strength between nodes, and $g_{0}$ is the transition rate of a node in the absence of cooperation.

The index $i$ runs from 1 to $N$, where $N$ is the total number of nodes of the complex network under study, implying that we have to run $N$ pairs of equations of the kind of Eqs. (4) and (5). The adoption of a fully connected network, or all-to-all condition, allows us to simplify the problem for analytical treatment. In fact, in that case, all the $N$ pairs of equations are identical to

$$
\begin{aligned}
& \frac{d}{d t} p_{1}(t)=-\frac{g_{12}(t)}{2} p_{1}(t)+\frac{g_{21}(t)}{2} p_{2}(t), \\
& \frac{d}{d t} p_{2}(t)=-\frac{g_{21}(t)}{2} p_{2}(t)+\frac{g_{12}(t)}{2} p_{1}(t),
\end{aligned}
$$

with

$$
g_{12} \equiv g_{0} \exp \left[K \frac{\left(N_{2}-N_{1}\right)}{N}\right]
$$

and

$$
g_{21} \equiv g_{0} \exp \left[K \frac{\left(N_{1}-N_{2}\right)}{N}\right] .
$$

Since it must be that $p_{1}(t)+p_{2}(t)=1$, it is convenient to reduce the pair of Eqs. (8) and (9) through a single equation for the probability difference,

$$
\Pi(t) \equiv p_{1}(t)-p_{2}(t)
$$

which, after simple algebra, is shown to read

$$
\frac{d}{d t} \Pi=\frac{\left(g_{21}-g_{12}\right)}{2}-\frac{\left(g_{21}+g_{12}\right)}{2} \Pi .
$$

It is important to stress that the equality

$$
\frac{N_{1}-N_{2}}{N}=\Pi
$$

holds true only in the limiting case $N=\infty$. In the more practical case where $N<\infty$, we have to adopt

$$
\frac{N_{1}-N_{2}}{N}=\Pi+f(t),
$$

where $f(t)$ is a random fluctuation, with intensity proportional to $1 / \sqrt{N}$, that is incurred by representing the mean field of the network as a probability difference. As a consequence it can be made arbitrarily small by increasing the number of interacting units. Plugging Eq. (15) into Eq. (13) we obtain

$$
\frac{d}{d t} \Pi=g_{0} \sinh [K(\Pi+f)]-g_{0} \Pi \cosh [K(\Pi+f)] .
$$

In the limiting case $N \rightarrow \infty, f=0$ and Eq. (16) generates a phase transition with the critical value

$$
K_{c}=1 \text {. }
$$

Considering $N$ large but finite and making an expansion of Eq. (16) to the lowest order nonvanishing contributions of $\Pi$ and $f$, we obtain

$$
\frac{d}{d t} \Pi=-\frac{g_{0}}{3} \Pi^{3}+g_{0} f
$$

at the critical point $K_{c}$. For notation convenience, we let $\Pi=x$ and write this nonlinear Langevin equation in the form

$$
\frac{d}{d t} x=-\gamma x^{3}+\xi(t)
$$

where

$$
\gamma \equiv \frac{g_{0}}{3}
$$

and

$$
\xi(t) \equiv g_{o} f(t) .
$$


Away from the critical point, the expansion of Eq. (16) gives a dominant linear friction term $-\gamma x$, with $\gamma \approx g_{0}|K-1|$, that is independent of $N$, and we recover an ordinary Langevin equation. A marked signal of criticality, then, is the vanishing of this linear term, leading to strongly nonlinear behavior that creates a subtle connection between the fluctuations and dissipation, providing a good example of an important effect noticed earlier by the authors of Ref. [23].

\section{RENEWAL NON-POISSON FLUCTUATIONS}

We notice that the stochastic Eq. (19) can be interpreted as describing the overdamped motion of a particle in the quartic potential

$$
U(x)=\frac{1}{4} \gamma x^{4}
$$

under the influence of a random noise. The Fokker-Planck equation for the probability distribution function (pdf) $p(x, t)$ corresponding to Eq. (19) reads

$$
\frac{\partial}{\partial t} p(x, t)=\left(\gamma \frac{\partial}{\partial x} x^{3}+D \frac{\partial^{2}}{\partial x^{2}}\right) p(x, t),
$$

yielding the equilibrium distribution

$$
p_{e q}(x)=\frac{1}{Z} \exp \left[-\frac{U(x)}{D}\right]
$$

where

$$
Z \equiv \int_{-\infty}^{+\infty} \exp \left[-\frac{U(x)}{D}\right] d x
$$

With simple dimensional arguments we find for the equilibrium second moment the relation

$$
\left\langle x^{2}\right\rangle_{e q} \propto\left(\frac{D}{\gamma}\right)^{\frac{1}{2}} .
$$

This means that the fluctuation $x$ spends most of its time in the interval

$$
-L<x<L,
$$

where

$$
L \equiv\left(\frac{D}{\gamma}\right)^{\frac{1}{4}}
$$

This expression allows us to establish with precision how to get rid of the border condition dictated by the probabilistic nature of $\Pi$. Since $-1 \leqslant \Pi \leqslant 1$, and the fluctuation $x$ is nothing but $\Pi$ expressed with a different notation suggesting it to be the coordinate of a Brownian particle, it is evident that we have to set the number of units $N$ large enough to satisfy the constraint

$$
D \ll \gamma .
$$

We assume that system's preparation is done at time $t=0$ with the initial condition $x=0$. We set $K=1$, thereby activating the stochastic Eq. (19). Note that the time scale of the process under study is determined as follows. We set the minimal time step for the solution of the master equation, $\Delta t=1$. This restricts us to selecting the number of events per unit of time $g_{0} \ll 1$. At short times the influence of the friction term $-\gamma x^{3}$ can be neglected and the second moment increases linearly in time,

$$
\left\langle x^{2}(t)\right\rangle=2 D t .
$$

To estimate the time $T_{e q}$ it takes for the system to reach equilibrium, we assume

$$
T_{e q} \approx \frac{\left\langle x^{2}\right\rangle_{e q}}{D}
$$

which through Eq. (26) leads to

$$
T_{e q} \propto\left(\frac{1}{\gamma D}\right)^{\frac{1}{2}} .
$$

We are in a position to address an issue closely related to the theoretical discussion made some years ago by Margolin and Barkai [24] to extend the Kramers approach to diffusion reactions [25] to the case where the reactant well is separated from the product well by a shallow barrier. In this case, the waiting time distribution density in the two states, thought of as the "dark" and the "light" states of the BQD intermittency, becomes the inverse power law $\psi(t)$ of Eq. (1) with $\mu=1.5$. We note that when $K>1$, the DM model has two equilibrium states corresponding to the bottoms of a double-well potential [26]. At criticality the barrier disappears, resulting in the potential of Eq. (22), generating the expected [24] inverse power law waiting time distribution over an extended time range. The same property was found in Ref. [15]. Here we recover this result analytically with the additional explanation of why the inverse power law is truncated at large times.

In fact, the population at $x=0$ depends on the recrossings, leading us to

$$
p(0, t) \propto \sum_{n=1}^{\infty} \psi_{n}(t),
$$

where $\psi_{n}(t)$ is the probability density for the $n$th return to the origin at time $t$. In the transient regime $t \ll T_{e q}$,

$$
p(x, t)=\frac{1}{\sqrt{4 \pi D t}} \exp \left(-\frac{x^{2}}{4 D t}\right),
$$

making $p(0, t) \propto 1 / t^{0.5}$. By Laplace transforming Eq. (33) it is possible to establish a connection between $\mu$ of Eq. (1) and the diffusion scaling index 0.5 of Eq. (34), yielding $\mu=1.5$. It is straightforward to prove with the same Laplace transform technique that for $t \gg T_{e q}$,

$$
\psi(t)=R \exp (-R t)
$$

with

$$
R \propto \frac{1}{Z}
$$

where $Z$ is given by Eq. (25). The numerical results of Fig. 1 confirm, in the survival probability for the return to the origin, a power law behavior that is truncated by an exponential at long times. The important conclusion of the theoretical and numerical analyses is that to make the inverse power law infinitely extended it is necessary to send the number of interacting units $N$ to infinity with the system at criticality.

Let us now discuss to what an extent the cooperative model we are using to explain intermittent fluorescence fits 


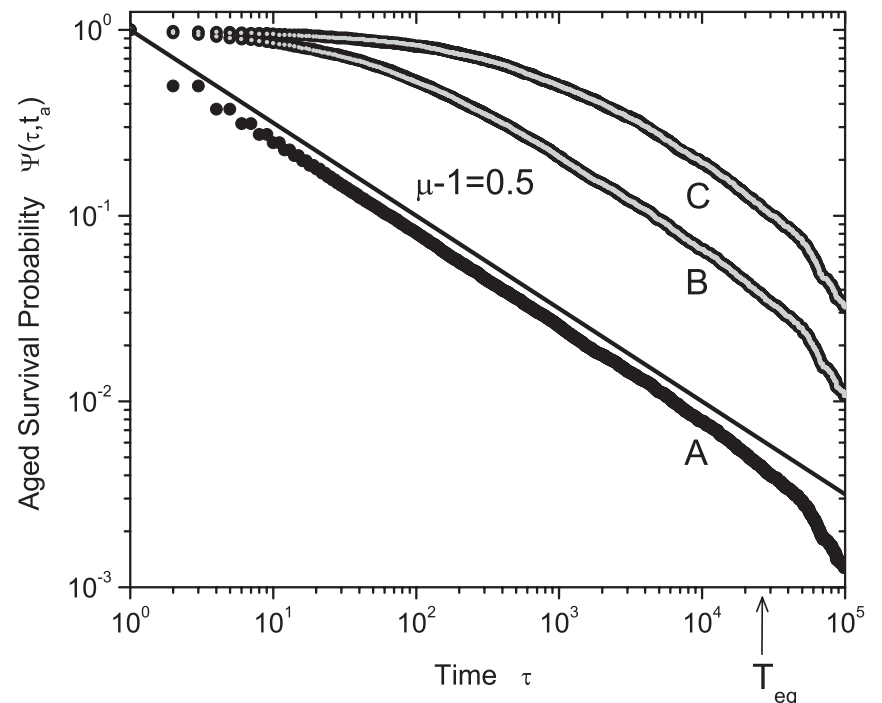

FIG. 1. Aged survival probability for the return to the origin of the fluctuation $x$ at the critical point $K=1$ with $g_{0}=0.01, N=1000$, and ages $t_{a}=0(\mathrm{~A}), t_{a}=100(\mathrm{~B})$, and $t_{a}=1000(\mathrm{C})$. In the absence of aging there is a power law with the index $\mu-1=0.5$ that is truncated by an exponential at $T_{e q}=2.6 \times 10^{4}$. In the presence of aging the survival probabilty decay becomes slower. The gray curves (lying directly on top of the black curves in (B) and (C)) represent the aging experiment performed on shuffled waiting time sequences. Their correspondence with the unshuffled black curves confirms that the aging is renewal.

the conditions of ergodicity breakdown that have triggered the recent advances in the field of statistical physics [7-10]. To shed light into this issue, let us imagine that the laminar region between two consecutive origin recrossings is assigned either the value 1 or -1 , according to a coin tossing prescription. This is equivalent to replacing the fluctuation $x(t)$ with a dichotomous fluctuation $\zeta(t)$. This dichotomous fluctuation has a nonstationary correlation function that depends on the time difference $t_{2}-t_{1}>0$ as well as the initial time $t_{1}$, thereby yielding $\left\langle\zeta\left(t_{2}\right) \zeta\left(t_{1}\right)\right\rangle=\Phi_{\zeta}\left(\tau, t_{a}\right)$, with $\tau \equiv t_{2}-t_{1}$ and $t_{a}=t_{1}$. As a typical behavior of complex systems, increasing $t_{a}$ (system's age) has the effect of making the correlation of the dichotomous fluctuation decay slower. To prove this important property, let us adopt the prescription of Ref. [6], resting on the study of the waiting time sequence $\tau_{1}, \tau_{2}, \ldots$, where $\tau_{i}=t_{i}-t_{i-1}$. As an improved version of this procedure we compare the original sequence to another sequence obtained from the original by randomly shuffling the waiting times. For $t_{a}=0$ the two sequences have the same waiting time distribution density, and consequently the same survival probability. The aged survival probability, or equivalently the aged correlation function $\Phi_{\zeta}\left(\tau, t_{a}\right)$ of the dichotomous fluctuation, is determined by means of a moving time window of size $t_{a}>0$, with the left end located on the time of occurrence of an event. We evaluate the time distance between the right end of the window and the next event. The corresponding aged waiting time distribution is normalized and its survival probability is $\Psi\left(\tau, t_{a}\right)$. If the two sequences, the original and the shuffled one, generate the same $\Psi\left(\tau, t_{a}\right)$, we conclude that the process is renewal. Figure 1 illustrates the results of this numerical analysis. We see that it is a non-Poisson renewal process. In fact, a Poisson process yields no aging, while the process studied in Fig. 1 generates a doublet of two coinciding aged survival probability functions. Furthermore, given that the survival probability for $t_{a}=0$ is a perfect inverse power law, we conclude that in the time region $t<T_{e q}$ the dichotomous fluctuation $\zeta(t)$ shares the properties of BQD intermittence that have created in the last few years an intensive search for proper statistical treatments of nonergodic renewal processes.

Note that this numerical experiment corresponds to evaluating the nonstationary correlation function of $\zeta(t)$, which is a dichotomous process different from

$$
\eta(t)=\operatorname{sgn}[x(t)]
$$

The waiting time distribution density of $\zeta(t), \psi^{*}(t)$, is related to the waiting time distribution density of $\eta(t)$ by

$$
\psi^{*}(t)=\sum_{n=1}^{\infty} 2^{-n} \psi_{n}(t) .
$$

By Laplace transforming Eq. (38) using the notation $\hat{g}(s) \equiv$ $\int_{0}^{\infty} \exp (-s t) g(t) d t$, we obtain

$$
\hat{\psi}^{*}(s)=\frac{\hat{\psi}(s)}{2-\hat{\psi}(s)} .
$$

Let us assign to $\psi(t)$ the analytical form

$$
\psi(t)=(\mu-1) \frac{T^{\mu-1}}{(t+T)^{\mu}} .
$$

Using

$$
\hat{\psi}(s) \approx 1-\Gamma(2-\mu)(s T)^{\mu-1}
$$

for $s \rightarrow 0$, we obtain that $\psi^{*}(t)$ has the same form as $\psi(t)$ with

$$
T^{*}=2^{\frac{1}{\mu-1}} T,
$$

implying that in the ideal case of $T_{e q}=\infty$, the correlation function $\left\langle\eta\left(t_{1}\right) \eta\left(t_{2}\right)\right\rangle$ shares the same aging properties as $\Phi_{\zeta}\left(t_{1}, t_{2}\right)$. In conclusion, this dichotomous fluctuation is compatible with the extension of LR to nonergodic intermittent processes and is expected to obey the new FDT of Refs. [9,10].

\section{FOKKER-PLANCK APPROACH TO LINEAR RESPONSE: ANALYTICAL TREATMENT}

\section{A. Nonstationary linear response}

Traditional LR theory cannot be used to fully describe the dynamics of the DM model in the presence of an external force for the two following reasons. First, the preparation of the system with all the dichotomous units entering into either of the two possible states simultaneously at time $t=0$ creates an out-of-equilibrium condition in that the rate of events must decrease as time evolves. Succeeding the preparation, the system relaxes toward equilibrium for an extended amount of time that is dependent on the number of interacting units. This reason alone is not enough to warrant the necessity of an extension of traditional LR since for any value of cooperation that is not the critical value, the system is described by a linear Langevin equation with a response that has an exact analytical solution valid for any magnitude of the external 
field. The second important reason then is that at the critical point $K=1$, the system is described by a nonlinear Langevin equation, which is a source of aging in the response function during the relaxation process.

To study the influence of an external stimulus acting on the dichotomous units whose dependence in time is described by the function $F_{\text {ext }}(t)$ we can replace Eq. (15) with

$$
\frac{N_{1}-N_{2}}{N}=\Pi+f(t)+F_{\text {ext }}(t) .
$$

Making the same Taylor series expansion that led us to Eq. (19) we now obtain

$$
\frac{d}{d t} x=-\gamma x^{3}+\xi(t)+F(t)
$$

where $F(t)=g_{0} F_{\text {ext }}(t)$. The time evolution of the corresponding pdf is given by

$$
\frac{\partial}{\partial t} p(x, t)=\left[\mathcal{L}_{0}+\mathcal{L}_{1}(t)\right] p(x, t)
$$

where

$$
\mathcal{L}_{0} \equiv \gamma \frac{\partial}{\partial x} x^{3}+D \frac{\partial^{2}}{\partial x^{2}}
$$

and

$$
\mathcal{L}_{1} \equiv-F(t) \frac{\partial}{\partial x} .
$$

To evaluate $\langle x(t)\rangle$ in the presence of perturbation we adopt the conventional first-order expansion of Eq. (45), $p(x, t) \approx$ $p_{0}(x, t)+p_{1}(x, t)[11]$, yielding

$$
p_{1}(x, t)=\int_{0}^{t} d t^{\prime} e^{\mathcal{L}_{0}\left(t-t^{\prime}\right)} \mathcal{L}_{1}\left(t^{\prime}\right) p_{0}\left(x, t^{\prime}\right) .
$$

We assume that the system is prepared at $t=0$ with $p_{0}(x)=$ $\delta(x)$. Note that during the transient regime $p_{0}(x, t)$ coincides with $p(x, t)$ of Eq. (34). This distribution is symmetric about the origin and remains so also in the final equilibrium condition. Therefore the nonvanishing contribution to $\langle x(t)\rangle$ depends only on $p_{1}(x, t)$,

$$
\langle x(t)\rangle=\operatorname{Tr}\left\{p_{1}(x, t) x\right\},
$$

resulting in

$$
\langle x(t)\rangle=\int_{0}^{t} d t^{\prime} \operatorname{Tr}\left\{x e^{\mathcal{L}_{0}\left(t-t^{\prime}\right)} \mathcal{L}_{1}\left(t^{\prime}\right) p_{0}\left(x, t^{\prime}\right)\right\} .
$$

Through an integration by parts this can be written as

$$
\langle x(t)\rangle=\int_{0}^{t} d t^{\prime} \operatorname{Tr}\left\{\left(e^{\Lambda_{0}\left(t-t^{\prime}\right)} x\right) \mathcal{L}_{1}\left(t^{\prime}\right) p_{0}\left(x, t^{\prime}\right)\right\},
$$

where

$$
\Lambda_{0}=\mathcal{L}_{0}^{\dagger} \equiv-\gamma x^{3} \frac{\partial}{\partial x}+D \frac{\partial^{2}}{\partial x^{2}}
$$

is the operator adjoint to the operator of Eq. (46).

Typically $p_{0}=p_{0}(x)$ is an equilibrium pdf independent of time. This results in a stationary response function and the time convolution LR structure. But since the system we are considering is characterized by an extended out-of-equilibrium transient regime and we are interested in the LR at a generic time, we must take into account the time dependence of $p_{0}=$ $p_{0}(x, t)$ when $t<T_{e q}$. Operating on the transient regime pdf of Eq. (34) with Eq. (47) and substituting the result into Eq. (51) leads to

$$
\langle x(t)\rangle=\int_{0}^{t} d t^{\prime} \operatorname{Tr}\left\{\left(e^{\Lambda_{0}\left(t-t^{\prime}\right)} x\right) \frac{x}{2 D t^{\prime}} p_{0}\left(x, t^{\prime}\right)\right\} F\left(t^{\prime}\right) .
$$

After evaluating the trace we are left with

$$
\langle x(t)\rangle=\int_{0}^{t} d t^{\prime} \Phi_{x}\left(t, t^{\prime}\right) F\left(t^{\prime}\right),
$$

where response function is the correlation function of the $x$ fluctuation,

$$
\Phi_{x}\left(t, t^{\prime}\right)=\frac{\left\langle x\left(t-t^{\prime}\right) x\left(t^{\prime}\right)\right\rangle}{\left\langle x^{2}\left(t^{\prime}\right)\right\rangle} .
$$

We see that in general the correlation function may be nonstationary, and when the system is at equilibrium the correlation function becomes stationary and we recover the traditional LR.

A weakness in the derivation of the extension of LR to Eq. (54) is that the nonstationary correlation function of Eq. (55) is derived only in the specific case using the purely diffusional pdf of Eq. (34). There exists an intermediate asymptotic time regime in which friction plays an important role before the system reaches equilibrium. The analytical expression of the pdf $p_{0}(x, t)$ in this time regime is unknown, but we expect it will generate a response fitting closely to Eq. (54), and rest on the numerical work of Sec. V to develop this heuristic assumption further.

It is useful to note that for the linear Langevin equation,

$$
\frac{d}{d t} x=-\gamma x+\xi(t)
$$

the correlation function is given by

$$
\Phi_{x}\left(t, t^{\prime}\right)=e^{-\gamma\left(t-t^{\prime}\right)}
$$

and is stationary everywhere despite the presence of the transient regime caused by the system's preparation at $x(0)=$ 0 . In the presence of an external force Eq. (56) becomes

$$
\frac{d}{d t} x=-\gamma x+\xi(t)+F(t)
$$

The exact solution of Eq. (58) yields

$$
\langle x(t)\rangle=\int_{0}^{t} d t^{\prime} e^{-\gamma\left(t-t^{\prime}\right)} F\left(t^{\prime}\right)
$$

for the average response, which is in agreement with Eq. (54).

In the case of free diffusion the correlation function is given by

$$
\Phi_{x}\left(t, t^{\prime}\right)=1,
$$

with the average response

$$
\langle x(t)\rangle=\int_{0}^{t} d t^{\prime} F\left(t^{\prime}\right) .
$$

This result can also be recovered by sending the friction coefficient $\gamma$ to zero in the linear Langevin equation. Again the LR structure of Eq. (54) holds despite the out-of-equilibrium nature of the diffusion process. 


\section{B. Linear response ignoring the action of fluctuations}

Under the incorrect but insightful approximation that the relaxation is due solely to the action of friction, as is true in the case of linear friction $-\gamma x$,

$$
x(t)=\exp \left(\Lambda_{0} t\right) x \approx \frac{1}{\left(\frac{1}{x^{2}}+2 \gamma t\right)^{\frac{1}{2}}},
$$

namely, the solution of $d x / d t=-\gamma x^{3}$, we obtain the LR structure

$$
\langle x(t)\rangle=\int_{0}^{t} d t^{\prime} \chi\left(t, t^{\prime}\right) F\left(t^{\prime}\right),
$$

where

$$
\chi\left(t, t^{\prime}\right) \equiv \int_{0}^{\infty} p\left(x, t^{\prime}\right) \frac{2 \gamma}{\left[1+2 \gamma x^{2}\left(t-t^{\prime}\right)\right]^{\frac{3}{2}}} d x .
$$

Which is the meaning of the linear response function of Eq. (64)? We notice that in the transient regime this response function reads

$$
\chi\left(t, t^{\prime}\right)=\int_{0}^{\infty} \frac{\exp \left(-\frac{x^{2}}{4 D t^{\prime}}\right)}{\sqrt{4 \pi D t^{\prime}}} \frac{2 \gamma}{\left[1+2 \gamma x^{2}\left(t-t^{\prime}\right)\right]^{\frac{3}{2}}} d x .
$$

Furthermore, when $4 D t^{\prime}$ is large enough, but not so large as to reach equilibrium, we have

$$
\chi\left(t, t^{\prime}\right) \approx \sqrt{\frac{\gamma}{2 \pi D t^{\prime}\left(t-t^{\prime}\right)}}
$$

this expression being nothing but the derivative with respect to $t^{\prime}$ of the survival probability of age $t^{\prime}$ [27]

$$
\Psi\left(t, t^{\prime}\right)=\int_{0}^{t^{\prime}} d t^{\prime \prime} R\left(t^{\prime \prime}\right) \Psi\left(t-t^{\prime \prime}\right)
$$

with $R(t) \propto 1 / t^{2-\mu}$, and $\mu=1.5$ [28].

In conclusion, by ignoring the vital role played by the fluctuations in the relaxation of $x(t)$ in the presence of nonlinear friction, we have shown that in an asymptotic time region where the final equilibrium is not yet perceived the linear response function $\chi\left(t, t^{\prime}\right)$ is given by

$$
\chi\left(t, t^{\prime}\right)=\frac{d}{d t^{\prime}} \Psi\left(t, t^{\prime}\right),
$$

where $\Psi\left(t, t^{\prime}\right)$ is the survival probability of age $t^{\prime}$. In the case where $T_{e q}=\infty$, this prescription holds true forever, implying a perennial departure from the ordinary prescription [11] based on the Onsager remarks that equilibrium corresponds to an infinitely aged condition where the infinitely aged survival probability becomes identical to the stationary equilibrium correlation function. Notice that Eq. (68) is a form of LR that has been used for the first time to prove that complex systems exchange information through the new principle of complexity management [13].

According to this principle the system does not respond to a stimulus that is not complex. The simplest case of a noncomplex stimulus is the harmonic signal

$$
F_{\text {ext }}(t)=\epsilon \cos (\omega t) .
$$

Adopting the exact solution for the response function of Eq. (65) yields

$$
\langle x(t)\rangle=\int_{0}^{t} d t^{\prime} \frac{\exp \left[\frac{1}{16 \gamma D\left(t-t^{\prime}\right) t^{\prime}}\right]\left(K_{1}\left[\frac{1}{16 D \gamma\left(t-t^{\prime}\right) t^{\prime}}\right]-K_{0}\left[\frac{1}{16 D \gamma\left(t-t^{\prime}\right) t^{\prime}}\right]\right)}{32 \sqrt{2 \pi \gamma}\left[D t^{\prime}\left(t-t^{\prime}\right)\right]^{3 / 2}} \epsilon \cos \left(\omega t^{\prime}\right),
$$

whereas the same approximation as that yielding Eq. (66) generates the analytical expression

$$
\langle x(t)\rangle=\epsilon \sqrt{\frac{\gamma \pi}{2 D}} J_{0}\left(\frac{\omega t}{2}\right) \cos \left(\frac{\omega}{2} t\right),
$$

implying the slow decay of $\langle x(t)\rangle$ in the long-time limit and consequently the insensitivity of the system to harmonic stimuli in the same time limit.

\section{JOINT ACTION OF NONLINEARITY AND FLUCTUATIONS IN THE RESPONSE TO HARMONIC PERTURBATION: NUMERICAL TREATMENT}

\section{A. Nondichotomous response}

In this section we show thanks to numerical calculations that the results of Sec. IV B, although insightful for understanding the recent extension of LR to nonergodic dichotomous processes, do not have any practical relevance when considering the response of the nondichotomous fluctuating variable $x$ of the DM model with finite $N$. In fact, increasing $N$ has the twofold effect of extending the transient regime virtually to infinity while making the fluctuation intensity of $x$ vanish. This is a property inherent in the recursion to the origin where, as shown in Fig. 1, the power law regime is valid in the region $t<T_{e q}$ and exponentially truncated at $t \approx T_{e q}$.

Due to the out-of-equilibrium preparation, the correlation function of the system at criticality is nonstationary in the transient regime and can be expressed in terms of its age as $\Phi_{x}\left(\tau, t_{a}\right)$ with $\tau=t-t^{\prime}$ and $t_{a}=t^{\prime}$. The aging behavior of the nonstationary correlation function is illustrated in Fig. 2 . In the limiting case $t_{a} \gg T_{e q}$ an exponentially decaying stationary equilibrium correlation function $\Phi_{e q}(\tau)$ is recovered and a nonaging behavior with it.

According to the demonstration at the end of Sec. IV A the correlation function of the linear Langevin equation is stationary everywhere regardless of the system's preparation. To see aging in the correlation function then, it is necessary to be at criticality where the fluctuations are nonlinear. Comparing to the aged survival probability shown in Fig. 1, which is equivalent to correlation function $\Phi_{\zeta}$, it becomes evident that the correlation function of the nondichotomous $x$ fluctuation ages differently than that of the dichotomous $\zeta$ 


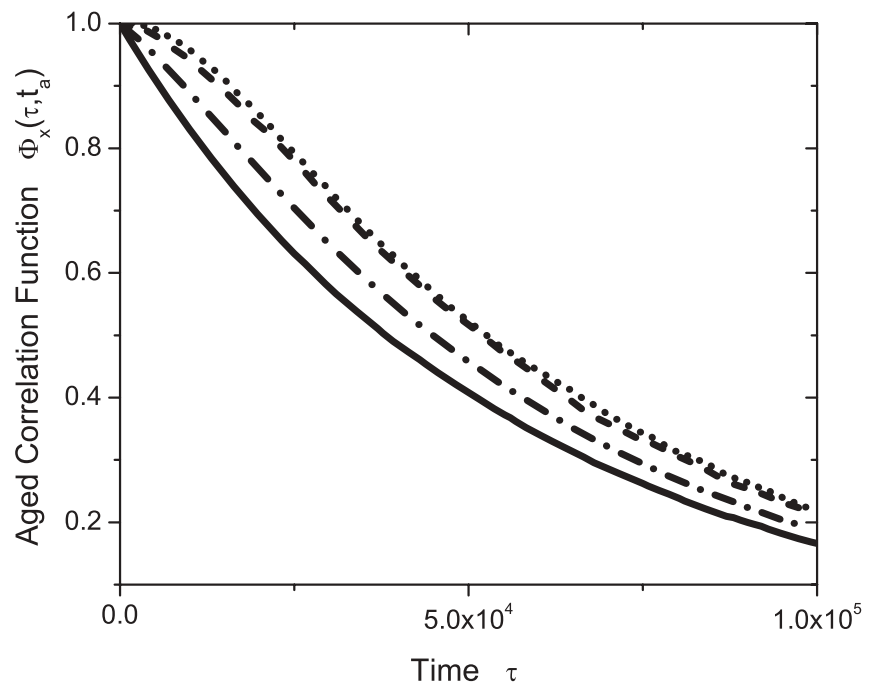

FIG. 2. Aged correlation function for the fluctuation $x$ at the critical point $K=1$ with $g_{0}=0.01, N=1000$, and ages $t_{a}=10^{2}$ (dot), $t_{a}=10^{3}$ (dash), $t_{a}=10^{4}$ (dash dot), and $t_{a}=10^{5}$ (solid). For ages $t_{a}<T_{e q}$ the correlation function is nonstationary (note: $T_{e q}=2.6 \times 10^{4}$ ). For ages $t_{a} \gg T_{e q}$ the aged correlation function becomes stationary and equivalent to the exponentially decaying equilibrium correlation function.

fluctuation. The survival probability decays more slowly with increasing age while the correlation function $\Phi_{x}$ decays more rapidly, although both tend toward an exponential behavior in the equilibrium time limit. Therefore we expect the response of the $x$ fluctuation to depart from the predictions of the ordinary LR for stationary processes, as well as the nonergodic FDT which applies to dichotomous fluctuations and relies on the derivative of the aged survival probability as its response function.

By replacing Eq. (19) with the approximate linearized expression

$$
\frac{d}{d t} x=-\Gamma x+\xi(t)
$$

where

$$
\Gamma=\gamma\left\langle x^{2}\right\rangle_{e q},
$$

it is possible to generate a relaxation process compatible with $T_{e q}$ of Eq. (32). As far as the response at criticality to an external perturbation is concerned, we solve numerically the perturbed nonlinear Langevin equation, Eq. (44). To see the effect of criticality we make a comparison to the numerical solutions of perturbed linear Langevin equations that correspond to $K$ subcritical and supercritical. Figure 3 shows the results.

We note that in the diffusional transient regime, which at criticality is much more extended in time than that generated by the linear Langevin equation, Eq. (44) becomes

$$
\frac{d}{d t} x=\xi(t)+F(t)
$$

yielding the average response

$$
\langle x(t)\rangle=\int_{0}^{t} d t^{\prime} F\left(t^{\prime}\right),
$$

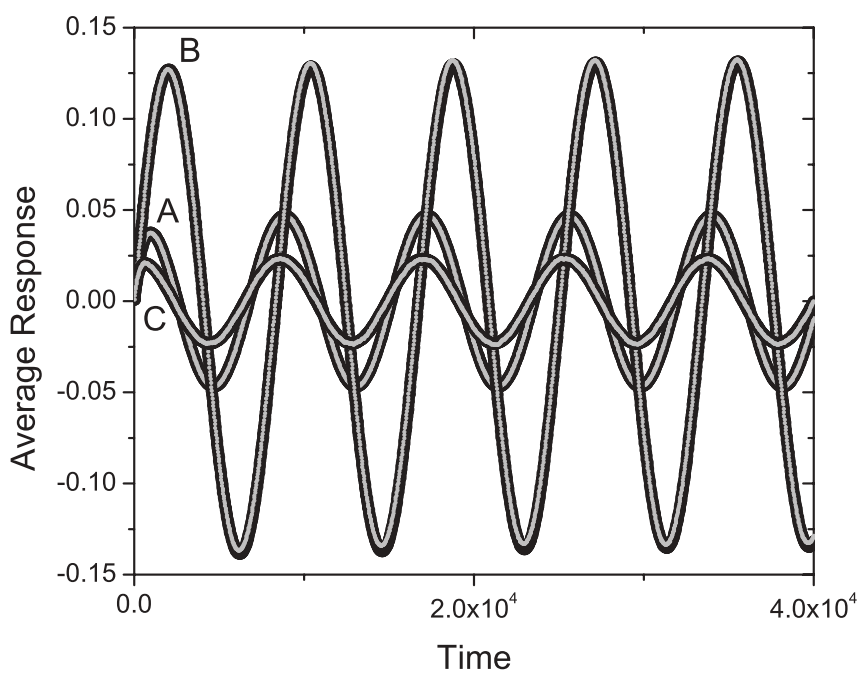

FIG. 3. Average response $\langle x(t)\rangle$ to a harmonic perturbation of intensity $\epsilon=0.01$ and frequency $\omega=7.5 \times 10^{-4}$ in the subcritical $K=0.8$ (A), critical $K=1.0$ (B), and supercritical $K=1.2$ (C) cases when $N=1000$ and $g_{0}=0.01$ (note: $\Gamma=3.8 \times 10^{-5}$ ). In each case the numerical solutions (gray) lie directly on top of the theoretical solutions (black) which are exact solutions to the perturbed linear Langevin equation. The response at criticality fits well with the linearization approximation for $\omega \gg \Gamma$.

which, in the case of the harmonic perturbation of Eq. (69) becomes

$$
\langle x(t)\rangle=\frac{g_{0} \epsilon}{\omega} \sin (\omega t) .
$$

It is convenient to make a connection with the predictions of stochastic resonance [29].

To this purpose we replace Eq. (44) with

$$
\frac{d}{d t} x=-\Gamma x+\xi(t)+F(t) .
$$

This approximation corresponds to assuming the network at criticality responds to perturbation according to the stationary correlation function

$$
\Phi_{e q}(\tau)=\exp (-\Gamma \tau)
$$

With this approximation it becomes legitimate to ignore the influence of the fluctuation $\xi(t)$ on the mean value of $x(t)$, and it is straightforward to prove that in the time region $t \gg 1 / \Gamma$ the response to the harmonic perturbation of Eq. (69) is given by

$$
\langle x(t)\rangle=\frac{g_{0} \epsilon}{\sqrt{\Gamma^{2}+\omega^{2}}} \cos (\omega t-\phi),
$$

where

$$
\tan \phi=\frac{\omega}{\Gamma}
$$

To establish a condition compatible with the numerical results of Fig. 3, we have to make the assumption that $\omega \gg \Gamma$, causing $\phi=\frac{\pi}{2}$, making Eq. (79) identical to Eq. (76).

To make a comparison with the subcritical and supercritical cases we use the perturbed linear Langevin equation, Eq. (58), with a friction coefficient $\gamma$, where $\gamma \approx g_{0}|K-1|$ and therefore $\gamma \gg \Gamma$. The form of response is left unchanged 


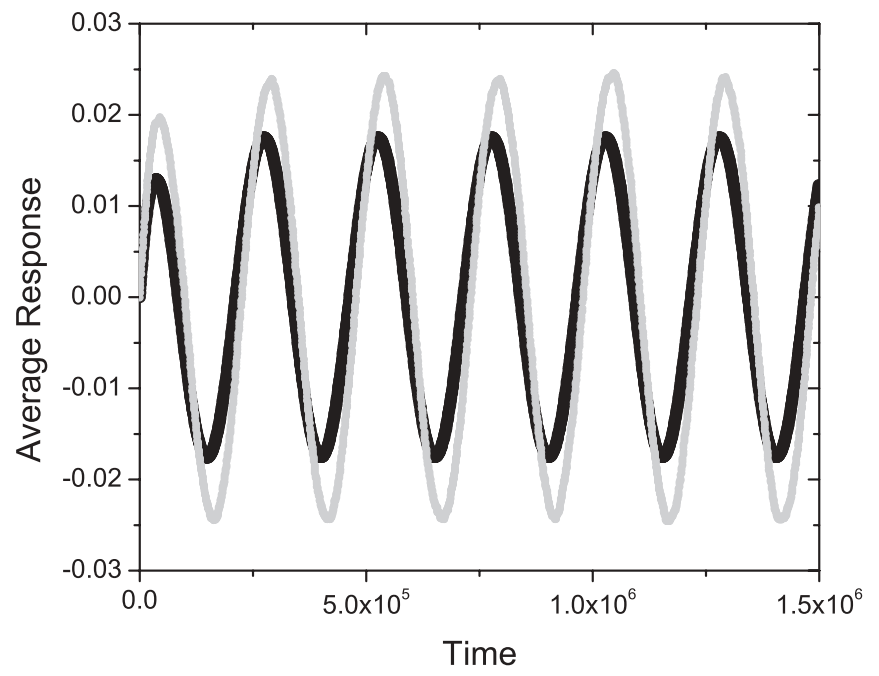

FIG. 4. Average response $\langle x(t)\rangle$ (gray) to a harmonic perturbation of intensity $\epsilon=8.0 \times 10^{-5}$ and frequency $\omega=2.5 \times 10^{-5}$ when $K=1, N=1000$, and $g_{0}=0.01$ (note: $\Gamma=3.8 \times 10^{-5}$ ). The theoretical curve (black) is the exact solution to the perturbed linear Langevin equation. A disagreement between the two curves shows that for sufficiently low perturbation frequencies, $\omega<\Gamma$, the response at criticality departs from the theoretical prediction given by the linearization approximation.

from Eq. (79), but due to a greatly reduced friction coefficient, at criticality the amplitude of the response is magnified as shown in Fig. 3, as long as the perturbation frequency $\omega$ is on the order of $\gamma$ or less. It should be noted that an even more significant increase in the amplitude of the response at criticality relative to subcritical and supercritical can be achieved by increasing $N$. This is due to the fact that when increasing $N$ the effective friction coefficient at criticality, $\Gamma \propto$ $1 / \sqrt{N}$, decreases, whereas for $K \neq 1$ the friction coefficient $\gamma$ remains unchanged.

An interesting frequency limit to study is $\omega \rightarrow 0$. In this low frequency limit, the linearization approximation, which was shown in Fig. 3 to be very accurate when $\omega \gg \Gamma$, departs from the results of numerical simulation, and we begin to see the nonlinear effects in action looking at Fig. 4. The amplitude of the response is even greater than that predicted by stochastic resonance in Eq. (79). This result cannot be explained by the conventional LR with the equilibrium correlation function as the response function, providing numerical support for the extension of LR made in Eq. (54) involving the nonstationary correlation function.

\section{B. Bridging the nondichotomous response and the dichotomous response}

For any choice of perturbation frequency the amplitude of the response of the nondichotomous fluctuation $x$ does not decay in time. This seems to be in conflict with the nonergodic FDT for dichotomous fluctuations where the amplitude is known to decay in time as $1 / t^{2-\mu}$. However, $\left\langle x^{2}(t)\right\rangle$ is free to grow in time in the transient regime while the dichotomous fluctuation $\eta(t)=\operatorname{sgn}[x(t)]$ fits the property $\left\langle\eta^{2}\right\rangle=1$ regardless of whether the system is at equilibrium or not. To be able to make a fair comparison to the predictions of the nonergodic FDT, we define

$$
\eta_{x}(t) \equiv \frac{x(t)}{\left\langle x^{2}(t)\right\rangle^{1 / 2}},
$$

generating a fluctuation that mimics the dichotomous property $\left\langle\eta_{x}^{2}\right\rangle=1$. Simply taking into account that during the transient regime $\left\langle x^{2}(t)\right\rangle=2 D t$, we immediately obtain

$$
\left\langle\eta_{x}(t)\right\rangle=\frac{\langle x(t)\rangle}{\left\langle x^{2}(t)\right\rangle^{1 / 2}} \propto \frac{1}{t^{0.5}} \epsilon \gamma \sin (\omega t),
$$

in accordance with the experimental observation [10] that the response of a nonergodic system to harmonic perturbation generates damped oscillations.

The second moment can be used as a bridge between the nonergodic FDT for dichotomous processes and the nonstationary LR used to describe the perturbation of the nondichotomous $x$ fluctuation. Figure 5 illustrates the connection between the response of the dichotomous fluctuation $\langle\eta(t)\rangle=\langle\operatorname{sgn}(x(t))\rangle$, which gives information only on the duration of the "light" and "dark" states, and $\left\langle\eta_{x}(t)\right\rangle$, which contains the full information of the nondichotomous process, but is divided by the square root of its time-dependent second moment. There is a good agreement outside of the short time limit, with the oscillations obtaining a constant amplitude after the system has reached equilibrium. The similarity between the decays of the two forms of response is difficult to observe since it requires an extended intermediate time window between the preparation of the system and $T_{e q}$, but potentially can be verified by taking the limit $N \rightarrow \infty$ so that the system virtually remains out of equilibrium forever.

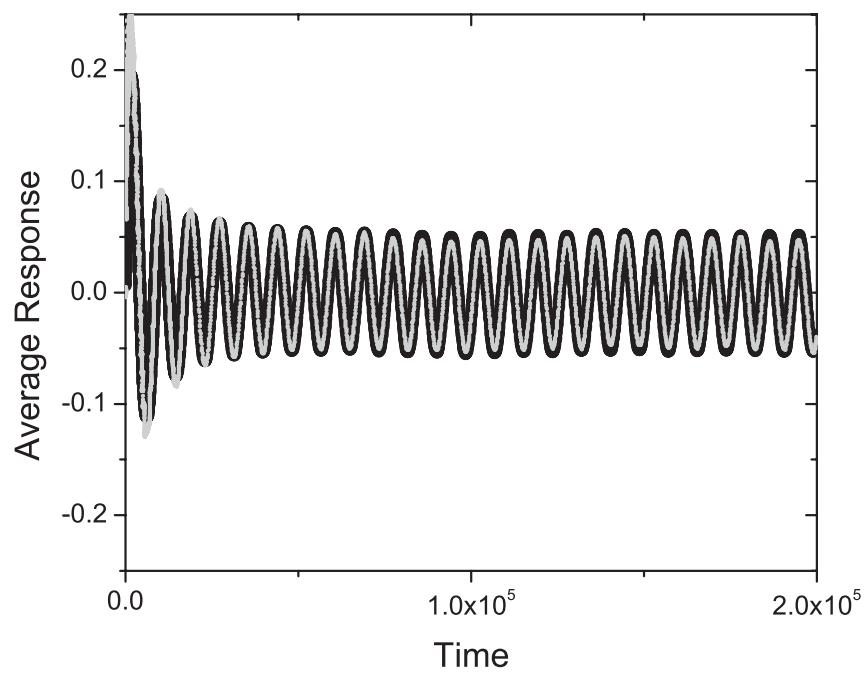

FIG. 5. Decaying average response $\left\langle\eta_{x}(t)\right\rangle$ (black) to a harmonic perturbation of intensity $\epsilon=2.0 \times 10^{-4}$ and frequency $\omega=7.5 \times$ $10^{-4}$ when $K=1, N=1000$, and $g_{0}=0.01$ (note: $T_{e q}=2.6 \times$ $\left.10^{4}\right)$. The damped oscillations in the transient regime are generated when dividing the undamped response $\langle x(t)\rangle$ by the square root of the unperturbed second moment, which is growing in time. The average response of the dichotomous fluctuation $\langle\eta(t)\rangle=\langle\operatorname{sgn}(x(t))\rangle$ (gray), where $x(t)$ is perturbed by the harmonic signal, is shown to decay in the same way as $\left\langle\eta_{x}(t)\right\rangle$ when disregarding the short time limit $t \rightarrow 0$. In the asymptotic time limit $t \gg T_{e q}$ the system reaches equilibrium with a constant second moment, resulting in undamped oscillations. 
The theoretical discussion of Sec. IV B has the merit of making clear that the nonstationary nature of the dichotomous fluctuation $\eta(t)$ is intimately related to the free diffusion process ensuing the system's preparation done at $t=0$. The rigorous treatment $[9,10]$ would lead to

$$
\langle\eta(t)\rangle=\int_{0}^{t} d t^{\prime} \chi\left(t, t^{\prime}\right) F\left(t^{\prime}\right),
$$

where

$$
\chi\left(t, t^{\prime}\right)=-\frac{d}{d t} \Psi\left(t, t^{\prime}\right)
$$

and $\Psi\left(t, t^{\prime}\right)$ is the aged survival probability [27]. Note that the nonergodic version of the FDT, using a phenomenological rather than dynamical argument, yields

$$
\chi\left(t, t^{\prime}\right)=\frac{d}{d t^{\prime}} \Psi\left(t, t^{\prime}\right),
$$

which, in the nonergodic case departs from Eq. (84). Although the principle of complexity management [30] is compatible with both the dynamical theory of Eq. (84) and the phenomenological theory of Eq. (85), the liquid crystal experiment of Ref. [10] shows that the response to harmonic perturbation fits the dynamical prescription and conflicts with the phenomenological prediction that with the perturbation of Eq. (69) would produce oscillation around a nonvanishing value slowly decaying to zero. In this sense the heuristic arguments leading to Eq. (82) seem to be compatible with the dynamical theory.

\section{CONCLUDING REMARKS}

The cooperative interaction between the units of the DM model in the case of all-to-all coupling generates a fluctuation $x(t)$ whose dynamics can be approximated by a Langevin equation that becomes nonlinear at the critical point. The out-of-equilibrium behavior ensuing the system's preparation generates at criticality a power law in the waiting time distribution for the return to the origin that extends over several orders of magnitude before being truncated by an exponential. If we were to consider fluctuations above the mean value as "light" states and fluctuations below as "dark" states we would recover a dichotomous fluctuation $\eta(t)=\operatorname{sgn}(x(t))$ with a renewal non-Poisson intermittent behavior that resembles BQD.

The power index $\mu=1.5$ is a well known property of the regression to the origin of a freely diffusing particle or one-dimensional random walker. However, in the case of the cooperation-induced fluctuation of the DM model used in this paper, this seems to be a coincidence rather than the true physical origin of this anomalous power index. The overdamped regression to equilibrium within the potential $U(x)$ of Eq. (22) generates the survival probability

$$
\Psi(t, x)=\left(\frac{1}{x}\right)\left(\frac{1}{1 / x^{2}+2 \gamma t}\right)^{0.5},
$$

which corresponds to $\mu=1.5$. Thus at criticality both free diffusion as well as dissipation involve the same power index. We note that the cooperative model adopted in Ref. [20] generates, as an effect of cooperation, $\mu \approx 1.3$. BQD have been known to display power law behavior with an index ranging anywhere from 1.2 to 2 , and it is expected that through modifications to the representation of cooperation it is possible to develop a model analogous to the DM model which reproduces this characteristic.

A striking result of this paper is that the FFP seems to emerge from a theoretical picture affording information only on the sequence of "light" and "dark" states, namely, from a dichotomous representation ignoring the complex details of the $x$ fluctuation. If these apparently complex details are taken into consideration then the nonergodic effects at criticality can be explained using a nonstationary LR as described by Eq. (54). The two forms of response are closely connected through the second moment of the $x$ fluctuation. By dividing the undamped response of the $x$ fluctuation by the square root of its second moment we can recover the decaying response behavior of the dichotomous fluctuation, allowing us to heuristically pass between the nonstationary LR and the nonergodic FDT.

By focusing our attention on the complete behavior of the nondichotomous fluctuation we have bypassed the ambiguity associated with external forces acting in the FFP picture. The main task then lies in determining the aging behavior of the correlation function and its effect on the response. Aging occurs only when the fluctuations are nonlinear, and this makes it difficult or impossible to write an exact analytical expression for the aged correlation function $\Phi_{x}\left(t, t^{\prime}\right)$. With numerical simulations we have shown that the stationary equilibrium correlation function can be used to accurately predict the response to harmonic perturbation in the high frequency limit, but in the low frequency limit the nonlinearity, and aging with it, play an important role in the response.

At criticality the duration of the out-of-equilibrium regime becomes dependent on the number of interacting units and is significantly extended in time. This is due to an $N$-dependent effective friction coefficient that has a magnitude much less than in the subcritical or supercritical cases. A direct consequence is that the response to a harmonic perturbation can be considerably greater in amplitude when a cooperative network is at criticality, a beneficial property for the transfer of information.

\section{ACKNOWLEDGMENTS}

M.B. acknowledges financial support from FONDECYT project No. 1110231. P.G. and A.S. warmly thank Welch and ARO for the financial support of this work through Grant No. B-1577 and Grant No. W911NF1110478, respectively.
[1] F. D. Stefani, J. P. Hoogenboom, and E. Barkai, Phys. Today 62, 34 (2009).

[2] T. D. Krauss and J. J. Peterson, Nat. Mater. 11, 14 (2012).
[3] C. Galland, Y. Ghosh, A. Steinbrück, M. Sykora, J. A. Hollingsworth, V. I. Klimov, and H. Htoon, Nature (London) 479, 203 (2011). 
[4] E. A. Riley, C. M. Hess, P. J. Whitham, and P. J. Reid, J. Chem. Phys. 136, 184508 (2012).

[5] X. Brokmann, J.-P. Hermier, G. Messin, P. Desbiolles, J.-P. Bouchaud, and M. Dahan, Phys. Rev. Lett. 90, 120601 (2003).

[6] S. Bianco, P. Grigolini, and P. Paradisi, J. Chem. Phys. 123, 174704 (2005).

[7] N. Korabel and E. Barkai, Phys. Rev. Lett. 108, 060604 (2012).

[8] S. Burov, R. Metzler, and E. Barkai, PNAS 107, 13228 (2010).

[9] P. Allegrini, M. Bologna, P. Grigolini, and B. J. West, Phys. Rev. Lett. 99, 010603 (2007).

[10] P. Allegrini, M. Bologna, L. Fronzoni, P. Grigolini, and L. Silvestri, Phys. Rev. Lett. 103, 030602 (2009).

[11] M. S. Green, J. Chem. Phys. 22, 398 (1954); R. Kubo, J. Phys. Soc. Jpn. 12, 570 (1957).

[12] I. M. Sokolov and J. Klafter, Chaos Solitions Fractals 34, 81 (2007); A. Weron, M. Magdziarz, and K. Weron, Phys. Rev. E 77, 036704 (2008).

[13] G. Aquino, M. Bologna, P. Grigolini, and B. J. West, Phys. Rev. Lett. 105, 040601 (2010).

[14] P. A. Frantsuzov, S. Volkán-Kacsó, and B. Jankó, Phys. Rev. Lett. 103, 207402 (2009).

[15] S. Bianco, E. Geneston, P. Grigolini, and M. Ignaccolo, Physica A 387, 1387 (2008).

[16] Th. Hartmann, V. I. Yudson, and P. Reineker, J. Lumin. 131, 379 (2011).
[17] B. I. Henry, T. A. M. Langlands, and P. Straka, Phys. Rev. Lett. 105, 170602 (2010).

[18] L. Reatto, Nat. Phys. 3, 594 (2007).

[19] A. Cavagna, A. Cimarelli, I. Giardina, G. Parisi, R. Santagati, F. Stefanini, and M. Viale, PNAS 107, 11865 (2010).

[20] F. Vanni, M. Lukovic, and P. Grigolini, Phys. Rev. Lett. 107, 078103 (2011).

[21] G. Werner, BioSystems 90, 496 (2007).

[22] D. Fraiman, P. Balenzuela, J. Foss, and D. R. Chialvo, Phys. Rev. E 79, 061922 (2009).

[23] A. V. Plyukhin and A. M. Froese, Phys. Rev. E 76, 031121 (2007).

[24] G. Margolin and E. Barkai, Phys. Rev. E 72, 025101(R) (2005).

[25] H. A. Kramers, Physica 7, 284 (1940).

[26] M. Turalska, M. Lukovic, B. J. West, and P. Grigolini, Phys. Rev. E 80, 021110 (2009).

[27] G. Aquino, M. Bologna, P. Grigolini, and B. J. West, Phys. Rev. E 70, 036105 (2004).

[28] W. Feller, An Introduction to Probability Theory and Its Applications, Vol. II (John Wiley \& Sons, New York, 1971).

[29] M. Lukovic, M. Ignaccolo, L. Fronzoni, and P. Grigolini, Phys. Lett. A 372, 2608 (2008); L. Gammaitoni, P. Hänggi, P. Jung, and F. Marchesoni, Rev. Mod. Phys. 70, 223 (1998).

[30] G. Aquino, M. Bologna, B. J. West, and P. Grigolini, Phys. Rev. E 83, 051130 (2011). 